\title{
Cable and Damper Vibration Studies
}

\begin{abstract}
A theoretical analysis of dampers and conductor vibration is presented in this paper and certain new methods of eliminating or reducing conductor vibration are discussed.
\end{abstract}

\author{
By \\ L. A. PIPES \\ NONMEMBER A.I.E.E.
}

California Institute of Technology, Pasadena

\section{$\mathbf{T}$}

$\mathrm{HE}$ interesting and important subject of mechanical vibration of transmission line conductors lends itself readily to a mathematical discussion if certain assumptions be made. Although these assumptions are not exactly true, they are quite close to the physical case, and produce results which should not be far in error. It is the purpose of this paper to present a fairly comprehensive analysis of several aspects of conductor vibration and of some of the methods which have been employed or suggested to lessen it.

\section{IMPERFECT FleXIBILITY}

In the discussion of transmission line vibrations it is customary to assume the cable tension to be of such great magnitude that it may be considered constant, and that the effect of rigidity may be neglected. In such an analysis the motion of the cable is described by the familiar wave equation in one dimension for small deviations from the static catenary curve produced by oscillations. The same equation is employed in the study of stringed instruments. If rigidity be taken into account, however, the equation of motion is:

$m \frac{\partial^{2} u}{\partial t^{2}}+E I \frac{\partial^{4} u}{\partial s^{4}}=T \frac{\partial^{2} u}{\partial s^{2}}+F(s, t)$

where

\footnotetext{
$u$ = lateral displacement of the cable from the catenary position of equilibrium, measured in a vertical plane and having a direction at right angles to the cable

$E=$ Young's modulus of elasticity for the material of the cable

$I=$ moment of inertia of the plane area cut by a plane normal to the cable about a diameter

$m=$ mass per unit length of cable

$T=$ tension, assumed constant throughout the span. This may be done with very little error for the usual span sag

$s=$ distance measured along the catenary curve as position of static equilibrium

$F=$ any arbitrary force per unit length acting along the conductor $t=$ time
}

\section{Traveling Waves}

A paper by W. B. Buchanan ${ }^{1}$ has stimulated a great deal of interest in the study of traveling waves along conductor spans. It may be profitable, therefore, to investigate the effect of conductor rigidity on such waves.

In the absence of any impressed or frictional forces equation 1 becomes

$m \frac{\partial^{2} u}{\partial t^{2}}+E I \frac{\partial^{4} u}{\partial s^{4}}=T \frac{\partial^{2} u}{\partial s^{2}}$

It is this equation which a traveling wave must satisfy. If $f$ is the frequency of the traveling wave, and the quantity

$x=\frac{16 \pi^{2} f^{2} m E I}{T^{2}}$

is so small that $x^{2}$ may be neglected in comparison with $x$, then the velocity of propagation $v$ of the wave is given by $v=\sqrt{T / m}$ and is thus independent of the frequency of the traveling wave. The criterion that $x$ be small is satisfied at frequencies of from 10 to 30 vibrations per second, the usual aeolian frequencies for the size of conductor ordinarily employed, if the size of cable is not very much greater than one inch in diameter. If the conductor is assumed to be a solid cylinder having a density $\rho$, an expression is obtained for $x$ in terms of $r$, the radius of the conductor: $x=\frac{4 \rho \pi^{4} E r^{6} F^{2}}{T^{2}}$, showing that the effect of rigidity is dependent upon the sixth power of the radius. At high frequencies or with large radii $x^{2}$ is not negligible compared to $x$. In such cases the effect of rigidity is marked and the law for the velocity of propagation is not a simple one. The velocity of propagation then depends upon the frequency and the other constants. The expression may be written

$v=\omega \sqrt{\frac{2 E I}{T}}\left[\sqrt{\frac{1}{\sqrt{1+\frac{4 E I m \omega^{2}}{T^{2}}}-1}}\right]$

where $\omega=2 \pi f$

The conclusions to be drawn from this are:

1. For the usual size of transmission conductors and for frequencies and tensions that are met in practice, the criterion that $x$ be small is met. In such cases the velocity of propagation is the same as that given by equation 1 if the term $E I$ involving rigidity is neglected. Hence, for the usual transmission line span, the effect of rigidity may be neglected for traveling waves.

2. For an impressed wave of a complex character, which may be resolved into a Fourier series, the high frequency components will not be propagated with a velocity independent of frequency and there will be marked distortion.

3 . Since the quantity $x$ varies as the sixth power of the radius of a sclid conductor, it may be seen that the effect of rigidity becomes

A paper recommended for publication by the A.I.E.E. committee on power transmission and distribution, and scheduled for discussion at the A.I.E.E. summer convention, Pasadena, Calif., June 22-26, 1936. Manuscript submitted Feb. 28, 1936; released for publication April 1, 1936.

The author wishes to acknowledge the assistance of R. W. Sorensen, S. S. Mackeown, and F. C. Lindvall, of the California Institute of Technology, A. E. Davison, of the Hydro-Electric Power Commission of Ontario, and D. M. Simmons, former chairman of the A.I.E.E. committee on power transmission and distribution.

1. For all numbered references, see list at end of paper. 
marked as soon as the conductor size passes a certain dimension. ( $x$ is quite small for conductors having a diameter of one inch or less.)

\section{Standing Waves on Rigid Conductors}

It is not surprising that there is an appreciable difference between the behavior of a perfectly flexible conductor and a rigid conductor. If standing waves exist, it is natural to find them distorted because of the effect of rigidity near the supports.

If equation 1 is solved under the assumption that no external forces exist, that $F=0$, and that absolute fixity exists at the 2 extremities of the span, the quantity $x$ is encountered again, and if it is assumed to be small, a solution for standing waves is possible. The effect of rigidity under this assumption is merely to disturb the nodal points, making their position a function of the distance from the points of support as well as the frequency of vibration. The nodal points are defined by a somewhat complex transcendental equation, but it is possible to calculate their position near the middle of the span. The general effect of rigidity appears to be to raise the frequency of all harmonics as given by the formula

$f=\frac{r}{2 l} \sqrt{\frac{T}{m}}\left[1+\frac{2}{l} \sqrt{\frac{E I}{T}}\right]$ where $r=1,2, \ldots$, etc.

and to shorten the wave length.

The effect of the small amount of rigidity present in a transmission line cable does not influence materially the propagation of traveling waves or the formation of standing waves; however, at higher harmonic frequencies the result becomes complicated. Fortunately the criterion that the quantity $x$ be small is met in a practical transmission line, and there is no need for a discussion of this complicated phenomenon.

\section{DAMPED VibRATIONS}

Since the effect of rigidity is not appreciable in a practical transmission line it is possible to study the effect of damping on the behavior of the vibrations by omitting the term due to rigidity in equation 1 . The physical law of damping actually followed by the cable is very complicated, and it cannot be formulated exactly; nevertheless, a law of damping that is approximately true and that can be analyzed mathematically can be assumed. Although the results are not exactly correct, they should give at least a qualitative view of the effects of damping.

- If it is supposed that the force $F$ in equation 1 is a force of viscous damping, or damping proportional to the transverse velocity of the cable,

$m \frac{\partial^{2} u}{\partial t^{2}}=T \frac{\partial^{2} u}{\partial s^{2}}-R \frac{\partial u}{\partial t}$

where the damping coefficient $R$ is assumed to be small.

\section{Traveling Waves With Damping}

In case a traveling wave on a conductor obeys the foregoing law of damping produced, say, by having one end of the span execute forced vibrations of a character $u=A \cos \omega t$ at $s=0$ it is found, on the supposition that $R$ is small, that

$u=A e^{-\frac{R s}{2} \sqrt{\frac{1}{m T}}} \cos \omega(t-s / a)$

Unless otherwise defined $a=\sqrt{T / m}$ in this and subsequent equations.

Equation 5 represents a traveling wave progressing in the positive $s$ direction with a velocity $a$ independent of the frequency. The wave undergoes a diminution in amplitude because of the attenuation factor, and the greater the tension and mass per unit length of the conductor, the smaller will be the attenuation. If the damping coefficient is very small, as it is usually, the amplitude diminishes in a manner proportional to the distance traveled; however, if $R$ is appreciable the damping is great, particularly at low values of tension.

It is interesting to observe that Buchanan ${ }^{1}$ found experimentally a greater attenuation of the traveling wave at low values of tension, and that the loss in amplitude is proportional approximately to the distance traveled. This effect can be seen from equation 5 , for if $R$ is quite small,

$\epsilon^{-\frac{R s}{2}} \sqrt{\frac{1}{m T}}=1-\frac{R S}{2} \sqrt{\frac{1}{m T}}$

approximately.

This shows that the loss in amplitude is proportional to the distance traversed. Hence, a rough experimental check is available for the assumed law of damping. The rate of decay is seen to be independent of the frequency, so that no distortion of a complex wave occurs.

\section{Standing Waves With Damping}

With the justifiable assumption that the damping coefficient $R$ is very small, it is possible to obtain a general solution of the damped wave (equation 4). This solution is:

$u=\epsilon^{-\frac{R t}{2 m}}\left[\phi_{1}(s-a t)+\phi_{2}(s+a t)\right]$

where $\phi_{1}$ and $\phi_{2}$ represent arbitrary functions of the argument $(s \pm a t)$. Physically $\phi_{1}$ is a wave of displacement traveling in the positive $s$ direction and $\phi_{2}$ is a wave traveling in the negative $s$ direction. The whole disturbance is subjected to a decay because of the term $\epsilon^{-\frac{R t}{2 m}}$. If a solution is sought to satisfy the restriction of fixity at the extremities of the span, on the supposition that $R$ is small,

$u=\epsilon^{-\frac{R t}{2 m}} \sum_{n=1}^{n=\infty}\left(A_{n} \sin \beta_{n} t+C_{n} \cos \beta_{n} t\right) \sin \frac{n \pi s}{l}$

where $A_{n}$ and $C_{n}$ are arbitrary constants depending upon the initial distortion of the cable from the position of equilibrium and

$\beta_{n}=\frac{1}{2} \sqrt{\frac{4 n^{2} \pi^{2} a^{2}}{l^{2}}-\frac{R^{2}}{m^{2}}}$ 
Fig. 1. The Stockbridge damper and an approximate equivalent
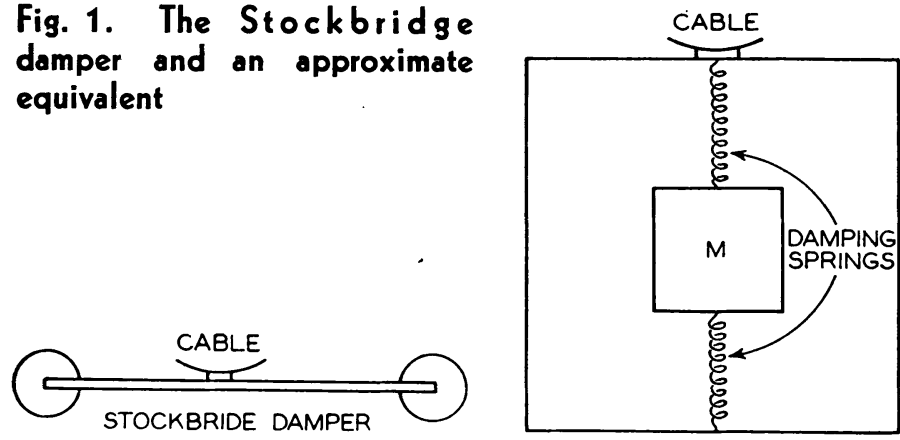

which is the solution for standing waves when damping is present. The position of the nodes is not changed by damping, but the whole disturbance undergoes a rapid decay in amplitude with time, and the periods of the various frequency components are increased by damping. The damping of standing waves, unlike the damping of traveling waves, is independent of the tension, and is greater for lower values of mass per unit length of the conductor. The powerful damping effect of the exponential term suggests the desirability of having a large damping coefficient $R$. If $R$ could be made large enough there would be no tendency for transient oscillations of the cable to arise.

\section{Forced Oscillations}

When a circular cylinder is placed in a moving fluid there is a tendency, in a real fluid such as air, to form a region of immobility behind the cylinder. Because of the shearing effect of the fluid stream on this dead wake, however, the fluid is set in rotation in the form of 2 eddies as shown in figure 3.

This dynamic system is most unstable, and when the stream attains a sufficient velocity, the vortexes formed in the wake no longer remain attached to the cylinder, but on reaching a sufficient size are carried downstream as if they were solid bodies.

Kármán and Rubach ${ }^{3}$ investigated the stability of the vortex system and Rayleigh ${ }^{4}$ gave an empirical formula for the frequency of detachment of these vortexes. Rayleigh's formula is:

frequency $=\frac{0.195}{D} \vee\left(1 .-\frac{20.1}{R}\right)$

where

$V=$ velocity of wind in feet per second

$D=$ diameter of cylinder in feet

$R=$ Reynold's number or the critical value of $V D / \nu$ at which eddies form expressed in suitable constants, and $\nu=\mu / \rho$ the kinematic coefficient of viscosity

$\mu=$ coefficient of viscosity of the fluid

$\rho=$ density of fluid

For the range of values of practical interest in transmission line vibrations use may be made of Relf and Ower's ${ }^{5}$ value for the frequency, $f=$ $0.185 V / D$, in which $V=$ velocity of wind relative to the cylinder in feet per second, and $D=$ diameter of cylinder in feet.

The problem is the finding of an expression for the transverse oscillatory force produced by the de- tachment of the vortexes and the drag force due to the wind. If use is made of the dynamically similar case discussed by Thom, ${ }^{6} F_{D}$ per square foot (drag force per square foot) $=K_{D} \rho V^{2}$, where $K_{D}$ is a constant varying but slightly with the velocity $V$, and $\rho$ is the density of the fluid.

For the maximum amplitude of the transverse force or pressure

$F_{T \text { per square foot }}=K_{T} \rho V^{2}$

The values of $K_{D}$ and $K_{T}$ vary with the velocity, but for the usual value of Reynold's number for flow of air around a cylinder having a diameter of about one inch, they may be taken to be approximately $K_{T}=0.45$ and $K_{D}=0.64$.

Using the average density of air, and converting units to the English system,

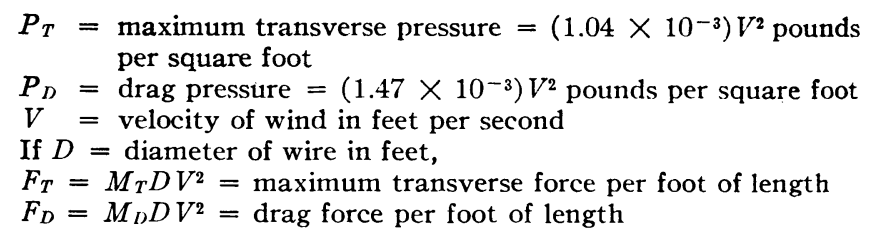

where the quantities $M_{T}$ and $M_{D}$ vary somewhat with the velocity of the wind but may be taken as reasonably constant throughout the usual range of wind velocities of from 0 to 10 miles per hour. For such a case the values $M_{T}=1 \times 10^{-3}$ and $M_{D}=$ $1.5 \times 10^{-3}$ produce the correct order of magnitude.

Having obtained this empirical expression for the maximum transverse force and drag force, the problem can be solved as follows: Assume that the transmission line span is subjected to a uniform horizontal wind having a velocity component $V_{W}$ at right angles to the length of the line. Assume further that the transmission line conductor is a cylinder suspended in space, acted on by the forces shown in figure 4 , and has a velocity $v$ transverse to the wind.

Now if the transverse force $F_{T}$ may be assumed as $F_{T}=M_{T} D V_{T}{ }^{2} \cos \omega t$ where $\omega=$ $\frac{(2 \pi)(0.185) V_{T}}{D}$ and $F_{D}=M_{D} D V_{T}^{2}$ components may be taken in the $u$ direction to obtain

$\sum F_{u}=F_{T} \cos \theta-F_{D} \sin \theta$ where $\theta=\tan ^{-1} \frac{v}{V_{W}}$

It may be assumed that in a practical case $V_{T}=$ $\sqrt{V_{W}{ }^{2}+v^{2}}=V_{W}$ approximately; therefore,

$\sum F_{u}=M_{T} D K_{W}{ }^{2} \cos \omega t-M_{D} D V_{W} v$

The action of the wind is seen to be that of a driving force $M_{T} D V_{W^{2}}$ cos $\omega t$ and a viscous damping force $M_{D} D V_{W}$.

Assuming the velocity of the wind to be reasonably constant throughout the span, it is permissible to substitute into the general equation of motion and obtain

$m \frac{\partial^{2} u}{\partial t^{2}}+R \frac{\partial u}{\partial t}=T \frac{\partial^{2} u}{\partial s^{2}}+M_{T} D V_{W^{2}} \cos \omega t-M_{D} D V_{W} \frac{\partial u}{\partial t}$

It will be noticed that the coefficient of the damping 
term contains 2 factors; $R$ due to the bending and twisting of the material, and $M_{D} D V_{W}$ due to the drag of the wind. ing,

On the assumption that there is negligible damp-

$\frac{2 \pi(0.185) V_{W} m}{D}>R+M_{D} D V_{W}$

and with the limitations imposed by the boundary conditions of fixity at the extremities, the solution of equation 10 is:

$u=\frac{23.8 M_{T} D^{3}}{w \sin K l}[\sin K s+\sin K(l-s)-\sin K l] \cos \omega t$

where

$w=$ weight of line, pounds per foot

$M_{T}$, a constant $=1 \times 10^{-3}$

$K=\omega \sqrt{\frac{v}{g T}}$

$\omega=\frac{1.16 V_{\mathrm{W}}}{D}$

$l=$ length of span, feet

$D=$ cable diameter, feet

$g \quad=32.2$ feet per second per second

$T=$ tension, pounds

$V_{W}=$ wind velocity, feet per second

This solution satisfies the conditions of fixity of the supports and represents a superposition of 2 waves, each having a wave length corresponding to the forced frequency, each with a node at one end, and with the proper compensating constant.

It must be realized, however, that the tentative assumption of negligible damping has been made. If

$\sin K l=0$ or $V_{W}=\frac{2.7 D_{n}}{l} \sqrt{T / m}$ and $n=1,2, \ldots$

a condition of resonance exists, and the amplitude apparently is infinite except at the nodal points $s=r l / n$.

However, if the small terms due to damping had not been rejected, sin $K l$ is not zero for any value of $K$, because $K$ then is complex and approximately equal to

$K=\frac{\omega}{a}\left[1-\frac{1}{2} j \frac{p}{\omega}\right]$

where

$p=\frac{g}{w}\left|R+M_{D} D V_{W}\right|$

$\omega=1.16 V_{W} / D$

$|\sin K l|_{\text {resonance }}=\sinh p l / 2 a$

and the vibration is slightly out of phase with the impressed force.

\section{ANALYSIS OF VibRATION DAMPER}

A mathematical analysis of the action of a damper of the Stockbridge ${ }^{2}$ type is possible if certain simplifying assumptions are made.
The usual Stockbridge damper consists of 2 masses supported at the ends of a rod and clamped to the conductor by means of parallel groove planks. The masses have weights of about 7 pounds each, and the distance between them is about 5 feet. In this analysis it is proposed to replace such a complex dynamic system by the simpler system of figure 1.

The approximation is permissible because the action of the rod is essentially that of a damped spring at whose ends are connected masses.

\section{Power Consumed by Damper}

Essentially, a damper is a device for extracting energy. Its action in suppressing vibration is due to its dissipation of the energy contained in the transient oscillations which the cable persists in following even after the action of the wind has stopped.

The average power consumed by the damper of figure 1 is:

$P_{\text {avg. }}=\frac{R M^{2} D^{2} \omega^{4}}{2\left[R^{2}+(\omega M-\mu / \omega)^{2}\right]}$

where

$D=$ displacement of cable at the damper

$\boldsymbol{R}=$ damping coefficient of the damper spring

$\mu=$ spring constant

$M=$ moving mass of the damper

$\omega=2 \pi f$

$f=$ frequency

Hence it is apparent that in the steady state the average amount of energy dissipated in the damper is much greater at the high frequencies; however, the higher frequencies have smaller amplitudes $D$ and the effect balances out.

The maximum power dissipated by the damper occurs at the resonant frequency $F_{R}=1 / 2 \pi \sqrt{\mu / M}$. It is $P_{\text {avg. }}=\frac{M^{2} D^{2} \omega_{R}^{4}}{2 R}$ where $\omega_{R}=2 \pi F_{R}$. It appears that if $R$ is quite small the damper will extract considerable energy at the resonant frequency. A damper should be designed, therefore, so that its

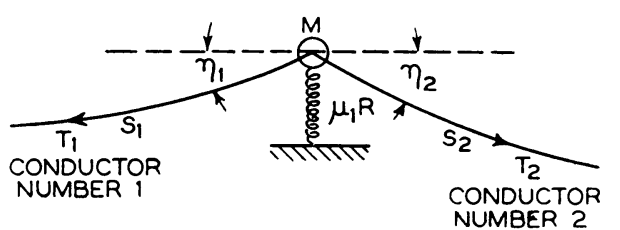

Fig. 2. System of forces acting on the end of a span with movable supports

natural or resonant frequency coincides with the frequency of the most troublesome vibration. If the damper contains several springs and masses, a band of frequencies may be damped. The dynamic displacement factor $D$ of the cable at the damper indicates that if the damper be placed at a node, the energy absorbed will be zero. Because of the irregularity of the wind and slight yielding of the supports, however, the nodes are not fixed points, and energy 
absorption always is taking place. The more fundamental action is that of a traveling wave on the damper which will be discussed later.

By maximizing the expression for the average power absorbed with respect to the various quantities the following results are obtained:

1. Optimum $R$ (other constants fixed)

$R^{2}=(\omega M-\mu / \omega)^{2}$

2. Optimum $M$ (other constants fixed)

$M=R^{2} / \mu+\mu / \omega^{2}$

3. Optimum $\mu$ (other constants fixed)

$\mu=\omega^{2} M$

The last condition indicates that the spring should be adjusted for resonance for best results, and suggests the greater flexibility of a damper whose spring constant or mass may be adjusted.

There is, of course, a danger that too great a flexibility of the spring may give rise to dangerous amplitudes and their corresponding destructive effect on the damper.

\section{Reaction of Damper on Conductor}

An expression for the dynamic reaction of a damper on an oscillating conductor shows rather clearly the mechanism by which energy is abstracted from the conductor. The expression is:

$F=\frac{\omega M D \sqrt{\mu^{2}+R^{2} \omega^{2}}}{\sqrt{R^{2}+(\omega M-\mu / \omega)^{2}}} \cos (\omega t-\theta)$

where

$$
\begin{aligned}
& \theta=\phi+\beta \quad \phi=\tan ^{-1} \frac{\omega M-\mu / \omega}{R} \\
& \beta=\tan ^{-1} \mu / \omega R
\end{aligned}
$$

in which the quantities have been defined previously. The point of support of the damper is experiencing the motion $u=D \cos \omega t$ and the static weight of the damper is neglected. With the condition that $\mu=\infty$ a correct expression is obtained for the reactive force due to a mass $M$ fixed to the conductor. The energy-abstracting action of the conductor is due to the phase displacement $\theta$. The reactive force is proportional to the amplitude of the motion and, at the resonant frequency, to the mass of the damper.

\section{Extremities Subject to Yielding}

An analysis of the behavior of standing waves may be carried out when the extremities are subject to yielding in a vertical direction if it is assumed that the ends of the cable are constrained to move in a vertical direction by the action of springs and masses attached to the ends. If the spring constants are denoted by $\mu$ and the masses by $M$, and if it is assumed that there is negligible damping in the conductor $(R=0)$, the solution is dependent upon the equation

$\frac{\partial^{2} u}{\partial t^{2}}=a^{2} \frac{\partial^{2} u}{\partial s^{2}}$ where $a=\sqrt{T / m}$ subject to the conditions:
1. At $s=0$
$M \frac{\partial^{2} u}{\partial t^{2}}+\mu u=T \frac{\partial u}{\partial s}$
2. At $s=l$
$M \frac{\partial^{2} u}{\partial t^{2}}+\mu u=-T \frac{\partial u}{\partial s}$
3. At $t=0$
$u=0$

The solution is:

$u=(A \sin r s+D \cos r s) \sin r a t$

in which

$\frac{A}{D}=\frac{\mu-M r^{2} a^{2}}{r T} \quad A=$ an arbitrary constant

$\tan r l=\tan 2 \theta \quad \theta=\tan ^{-1} \frac{\mu-M a^{2} r^{2}}{r T}$

The analysis of the general case thus leads to a somewhat complicated transcendental equation for the possible values of $r$. The accuracy can be

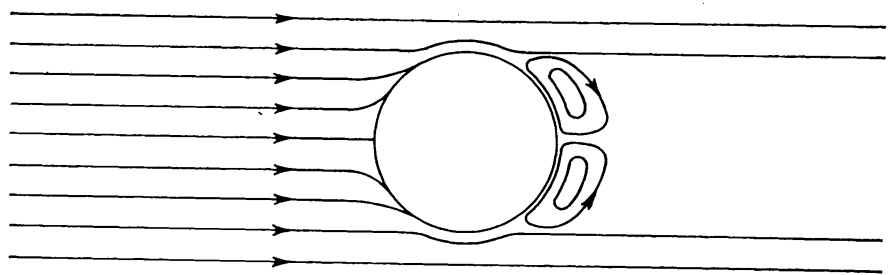

Fig. 3. Formation of vortexes behind a cylinder in a moving fluid

checked, however, by considering first whether it reduces to the correct solution for the rigidly fixed case. This can be done by placing $\mu=\infty$ and $M=0$. The result is :

$\tan \theta=\infty, \quad \theta=\pi / 2+2 s \pi$ and $s=0,1, \ldots$

$\tan 2 \theta=\tan (\pi+4 s \pi)=0=\tan r l$

therefore

$r=\frac{n \pi}{l}$ and $\frac{A}{D}=\infty$

and

$u=\sum_{0}^{\infty} A_{n} \sin \frac{n \pi s}{l} \sin \frac{n \pi a}{l} t$

This agrees with the solution obtained for rigid ends which may be obtained by elementary methods, and is the solution for standing waves.

\section{Slight Mobility of Supports}

By placing $M=0$ and letting $\mu$ be' great, the practical case of slight yielding at the supports is obtained, and the following expressions are applicable:

$f=$ frequency of vibration $=\frac{n a}{l}\left(1-\frac{2 T}{l_{\mu}}\right)$ 
$\frac{A}{D}=\frac{\mu}{r T}$ and $r=\frac{n \pi}{l}\left(1-\frac{2 T}{l_{\mu}}\right)$

by which it is indicated that the frequencies of the harmonics are slightly lowered. The ratio $A / D$, however, yields the important information that there are no nodes. This is evident from the observation that even though $A / D$ is large and $A$ is arbitrary, nevertheless $D$ is not zero, and from the equation

$u=(A \sin r s+D \cos r s) \sin r a t$

it appears that there are no true nodes although there are points at which the displacement $u$ is always small as a consequence of the smallness of $D$. Perhaps this conclusion explains the action of the damper at almost any point. The solution of the general case requires a graphical analysis of equation $19 b$, for which given values of the constants must be used.

\section{Reflection Coefficients}

The fundamental position of traveling waves in the theory of vibration suggests the value of an analysis of their reflection at points of discontinuity. The complete derivation of these equations is found in the appendix.

Reflection of Traveling Wave at End of Span Having Movable Supports. In this analysis the system of figure 2 is assumed. The span terminates on a mass $M$, which is given a degree of vertical freedom by a

\section{Fig. 4. Diagram of wind forces acting on a trans- mission line con- ductor}

$v=$ velocity of cylinder in $u$ direction as a result of its oscil. lation $\partial u / \partial t . \quad V_{w}=$ component of wind velocity acting in horizontal direction at right angles to the span. $V_{t}=$ velocity of wind relative to the moving cylinder

spring that is supposed to have a spring constant $\mu$ and a viscous resistance coefficient $R$. For generality it may be assumed that the 2 spans have the masses per unit length $m_{1}$ and $m_{2}$, respectively, and the tensions $T_{1}$ and $T_{2}$. It is found that for the size of cable ordinarily used (about one inch in diameter) and for frequencies of the order of magnitude of the objectionable frequencies present in transmission lines, the effect of rigidity in the reflection and transmission coefficients may be neglected, provided that the tensions are not such as to allow a sag exceeding $1 / 10$ of the span length.

General Reflection Coefficient. If the incident wave traveling along conductor number 1 of figure 2 be represented by the equation

$u_{I}=A \cos \omega\left(t+s_{1} / v_{1}\right)$ where $v_{1}^{\prime}=\sqrt{T_{1} / m_{1}}$
The reflected wave along conductor number 1 is given by

$u_{R}=\frac{A Q}{P} \cos \left[\omega\left(t-\frac{s_{1}}{v_{1}}\right)+\theta\right]$

where

$Q=\sqrt{\alpha^{2}+(\beta-\gamma)^{2}}$

$P=\sqrt{\alpha^{2}+(\beta+\gamma)^{2}}$

$\alpha=\omega^{2} M-\mu$

$\beta=\omega \sqrt{m_{1} T_{1}}$

$\gamma=\omega\left(\sqrt{m_{2} T_{2}}+R\right)$

$\theta=\theta_{1}-\theta_{2}$

$\theta_{1}=\tan ^{-1} \frac{\beta-\gamma}{\alpha}$

$\theta_{2}=\tan ^{-1}-\frac{(\beta+\gamma)}{\alpha}$

This complex expression may be simplified by the assumption that the adjacent spans are identical; that is, $m_{1}=m_{2}$ and $T_{1}=T_{2}=T$, then

$$
\begin{aligned}
\frac{Q}{P} & =\sqrt{\frac{\alpha^{2}+\omega^{2} R^{2}}{\alpha^{2}+\omega^{2}(R+2 \sqrt{m T})^{2}}} \\
& =\sqrt{\frac{\omega^{2}\left(M-\mu / \omega^{2}\right)^{2}+R^{2}}{\omega^{2}\left(M-\mu / \omega^{2}\right)^{2}+(R+2 \sqrt{m T})^{2}}}
\end{aligned}
$$

showing greater reflection at the higher frequencies. At the frequencies for which the spring and mass at the ends are in resonance,

$\omega=\sqrt{\mu / M}$ and $\frac{Q}{P}=\frac{R}{R+2 \sqrt{m T}}$

Since $R$ is small, it is apparent that there is a small amount of reflection at the resonant frequency.

\section{Transmission CoEfFicients}

If the supports are movable, a traveling wave in span 1 (figure 2) proceeds to span 2. The transmitted wave is defined by

$u_{\text {trans. }}=\frac{\delta_{1}}{\delta_{2}} \frac{A Q_{1}}{P} \cos \left[\omega\left(t-s_{2} / v_{2}\right)+\epsilon_{1}\right]$

in which

$$
\begin{aligned}
& P=\sqrt{\alpha^{2}+(\beta+\gamma)^{2}} \quad Q_{1}=2 \omega \sqrt{m_{1} T_{1}} \\
& \alpha=\omega^{2} M-\mu \quad \gamma=\omega\left(\sqrt{m_{2} T_{2}}+R\right) \\
& \delta_{1}=\cos \eta_{1} \\
& \delta_{2}=\cos \eta_{2} \\
& \epsilon_{1}=\pi / 2-\theta_{3} \quad \theta_{3}=\tan ^{-1} \frac{\alpha}{(\beta+\gamma)}
\end{aligned}
$$

For ordinary spans, the angles $\eta_{1}$ and $\eta_{2}$ are small, and the ratio $\frac{\delta_{1}}{\delta_{2}}$ may be taken as unity, if as before, the spans are identical. The expression $Q_{1} / P$ reduces to

$\frac{Q_{1}}{P}=\frac{2 \omega \sqrt{m T}}{\sqrt{\left(\omega^{2} M-\mu\right)^{2}+\omega^{2}(R+2 \sqrt{m T})^{2}}}$ 
where

$m_{1}=m_{2}=m$ and $T_{1}=T_{2}=T$

This expression reduces to zero if $\mu=\infty$ or the end is fixed. At resonance the expression is a maximum, showing that in this case there is a minimum of reflection and a maximum of transmission. If $M$ is appreciable the reflection coefficient becomes quite small at the higher frequencies.

Since both the reflection and transmission coefficients involve the frequency in a nonlinear manner, it is apparent that when a complex wave, which may be resolved into simple waves of several frequencies, impinges on a movable support it is partially reflected and partially transmitted in a very complex manner. Since the transmission coefficient is highest for the wave that is in resonance with the movable supports it might be possible in this manner to build up objectionable vibrations in several adjacent spans as a result of the discriminating action of the supports in favor of waves of that particular frequency.

\section{Reflection From DAMPer}

In an analysis of the action of a vibration damper it is of interest to observe the effect of a damper on a traveling wave. On the assumption that the frequency of vibration and the size of wire are such that the effects of rigidity may be neglected as discussed previously, and that the vibrations are of the small amplitudes

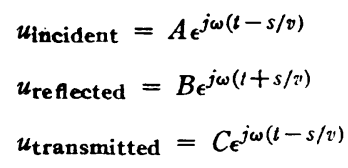

because of the linearity of the equations, only the real parts of the solutions are retained. If equation 18 is used for the dynamic reacting force of the damper the boundary conditions can be defined as usual, and the reflection and transmission coefficients may be obtained. Because of the complex character of the reactive force the expressions for these coefficients are quite involved.

\section{Reflection Coefficient}

If the incident wave is represented"by the equation $u_{t}=A \cos \omega(t-s / v)$ where $v=\sqrt{\frac{T}{m}}$

the reflected wave is defined by

$u_{R}=-\frac{A k}{V} \cos \left\{\omega(t+s / v)+\left(\epsilon-\beta_{1}\right)\right\}$

in which

$$
\begin{array}{ll}
\frac{K}{V}=\frac{1}{\sqrt{1-\frac{4 \omega^{2} R \sqrt{m T}}{z_{1}{ }^{2}}+\frac{4 z^{2} m T}{z_{1}{ }^{2} M^{2}}}} & \\
z=\sqrt{R^{2}+(\omega M-\mu / \omega)^{2}} & z_{1}=\sqrt{\omega^{2} R^{2}+\mu^{2}} \\
\epsilon=-(\phi+\beta) & \phi=\tan ^{-1} \frac{\omega M-\mu / \omega}{R}
\end{array}
$$

$$
\begin{aligned}
& \beta=\tan ^{-1} \frac{\mu}{\omega R} \\
& K=\frac{\omega M \sqrt{R^{2} \omega^{2}+\mu^{2}}}{\sqrt{R^{2}+(\omega M-\mu / \omega)^{2}}}
\end{aligned}
$$$$
\beta_{1}=\tan ^{-1} \frac{K \sin \epsilon+2 T \omega / v}{K \cos \epsilon}
$$

The quantities $M, R$, and $\mu$ are those defined previously for the damper. When the denominator of the expression for $K / V$ becomes imaginary, it is interpreted as a 90 degree further change of phase in the reflected wave. The complexity of this expression makes it difficult to analyze. Since the damping coefficient $R$ is small, however, it appears that the denominator of the expression for $K / V$ is approximately equal to unity if the frequency of the traveling wave is the same as the resonance frequency of the damper, thus, it appears that traveling waves having frequencies close to the resonance frequency of the damper are quite strongly reflected. Of course, perfect reflection does not occur in this case because of the viscous damping coefficient $R$ of the damper. Much information cannot be obtained from the above equations, however, without the use of numerical values for the constants. This reduction may be done in a practical case.

\section{ENERgy CONSIDERATIONS \\ of Traveling Waves}

Any vibration suppressing device must in some manner extract energy from the vibrating transmission line. On the hypothesis that the tension of the cable is great in comparison with its mass per unit length, an expression may be written for the average energy per unit length of the wire. The expression for a traveling wave of amplitude $A$ and of frequency $f=\omega / 2 \pi$ is :

$W=\frac{\text { energy }}{\text { length }}=\frac{1}{2} m \omega^{2} A^{2}$

This relation shows that the high frequency components of a complex traveling wave carry an appreciable amount of energy even if their amplitudes be small. Since the energy varies as the square of the amplitude, it is apparent that any factor that tends to increase the damping coefficient $R$ has a great effect in suppressing transient oscillations. This may be seen from the equation of damped traveling waves

$u=A \epsilon^{-\frac{R s}{2} \sqrt{\frac{1}{m T}}} \cos \omega(t-s / a)$

In case $R$ is appreciable the amplitude is reduced rapidly with distance-- Any factor such as interstrand friction, which tends to increase $R$, has great influence in absorbing energy and in mitigating vibration.

\section{EFFect of Wind Velocity}

The amplitude of the forced oscillation is dependent on $V_{W}$ in the following ways:

1. The angular frequency $\omega=1.16 V_{W} / D$ varies directly with the wind velocity. 
2. The imaginary part of $K$, or the part that prevents sin $K$ from going to zero and limits the amplitude at quasi-resonant values of $\omega$, increases with an increase of $\omega$ or of wind velocity. This is due to the wind velocity viscous damping term. See equation 11 .

3. The factor $M_{T}$ increases somewhat with wind velocities.

\section{Effect of Cable Constants}

The weight of the cable appears to lessen the damping effect of the wind and the damping factor $R$ of the material. An increase in the weight per unit length lessens the damping effect; however, the factor $w$ in the denominator of the expression for $u$ lessens the amplitude of the nonresonant vibrations.

The equation for $K$ shows the effective lessening of the damping caused by an increase in tension.

It may be seen that as long as damping is considered, or $K$ is taken as complex, there are no true nodes except at the ends. There are, of course, points of minimum and maximum amplitude, but they are not true nodes or antinodes. If the wind is of variable velocity, these false nodal points must continually shift their positions because of changing values of $\omega$. The amplitude appears to be a function of the cube of the diameter, suggesting that cables of large diameter are most prone to large amplitudes.

The fact that at the resonant frequency

$|\sin K l|_{\text {resonance }}=\sinh \left[\frac{l}{2} \sqrt{\frac{m}{T}}\left(R+M_{D} D V_{W}\right)\right]$

shows most clearly the influence of several line constants on the magnitude of the amplitude at resonance.

\section{Hollow Cables}

The Swiss engineer, Max Preiswerk, thas proposed a theory of a vibrationless cable. He proposes to have a hollow conductor inside of which is a steel core. The steel core is loose and at a different tension than the envelope. Preiswerk argues that in such a system 2 natural frequencies exist and one or the other is always opposing the establishment of all types of standing waves resulting from an accumulation of traveling waves. The situation, he argues, is similar to that of 2 coupled vibrating systems which, when coupled together, have a natural frequency differing from that of either system taken separately.

A mathematical analysis of this theory produces many interesting conclusions. Since a mathematical analysis of a loose core is impossible because of the discontinuous character of the motion, consideration . will be given to a system that is more responsive to analysis and less destructive in its action, for it does not allow the 2 conductors to bump together.

Assume that some material of an elastic character is inserted between the inner and outer cables. For the purpose of analysis, assume that this material produces an interaction between the cable and the core proportional to the difference of the absolute displacements at right angles to the lengths of the conductors. Assume also that the packing material absorbs energy in such a way as to produce viscous damping proportional to the relative velocities between the inner and outer cables.

Let all quantities with a subscript 1 refer to the inner cable, and all quantities with a subscript 2 refer to the outer hollow cable. Then let

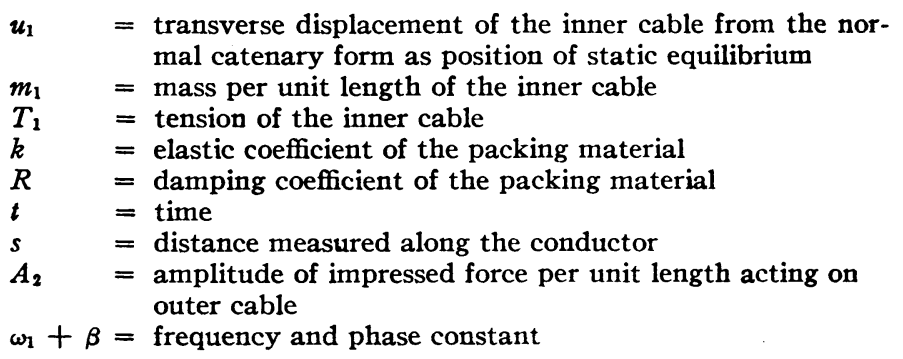

Under the assumption that the tension is practically constant throughout the span length, andishat the amplitudes of the transverse vibrations "are small, the equations of motion are:

$$
\begin{aligned}
& m_{1} \frac{\partial^{2} u}{\partial t^{2}}=T_{1} \frac{\partial^{2} u}{\partial s^{2}}+k\left(u_{2}-u_{1}\right)+R \frac{\partial}{\partial t}\left(u_{2}-u_{1}\right) \\
& m_{2} \frac{\partial^{2} u}{\partial t^{2}}=T_{2} \frac{\partial^{2} u}{\partial s^{2}}+k\left(u_{1}-u_{2}\right)+R \frac{\partial}{\partial t}\left(u_{1}-u_{2}\right)+ \\
& A_{2} \cos (\omega t+\beta s)
\end{aligned}
$$

Since the inner and outer conductors must hang in identical catenary curves in the static state, each static curve must be equal to $y=H / w \cosh (x w / H)$ in which $m=w / g$, and $g$ is the constant of acceleration due to gravity. To a great degree of accuracy for a taut cable $H=T$. The static curves must be identical so that there will not be a static stress, and the condition that

$\frac{T_{1}}{m_{1} g} \cosh \frac{x m_{1} g}{T_{1}}=\frac{T_{2}}{m_{2} g} \cosh \frac{x m_{2} g}{T_{2}}$

must be valid for all values of $x$. If, in particular, $x=0$ then $T_{1} / m_{1} g=T_{2} / m_{2} g$ or $T_{1} / m_{1}=T_{2} / m_{2}=a^{2}$. This equality satisfies all values of $x$.

\section{StANDING WAVES}

Eliminating $u_{1}$ from equations $22 a$ and $23 a$, placing $A_{2}=0$ for the freely vibrating case, and assuming a solution of the type $u=C \epsilon^{j(\omega t+\beta s)}$ produces the following solution for $u_{2}$ :

$$
\begin{aligned}
& u_{2}=\sum_{0}^{\infty}\left(A_{n} \sin \frac{\pi a n t}{l}+B_{n} \cos \frac{\pi a n t}{l}\right) \sin \frac{\pi n s}{l}+ \\
& \epsilon^{-R t / 2 m} \sum_{0}^{\infty}\left(C_{n} \sin \alpha_{n} t+D_{n} \cos \alpha_{n} t\right) \sin \frac{n \pi s}{l}
\end{aligned}
$$

in which

$$
\begin{array}{lr}
\frac{1}{m}=\frac{1}{m_{1}}+\frac{1}{m_{2}} \quad \alpha_{n}=\sqrt{\frac{k}{m}+\frac{a^{2} n^{2} \pi^{2}}{l^{2}}-\frac{R^{2}}{4 m^{2}}} \\
l=\text { span length } \\
A_{n}, B_{n}, C_{n}, D_{n}=\text { arbitrary constants }
\end{array}
$$

Use has been made of the fact that the supports are rigid and are nodal points. The first summation 
in the solution for $u_{2}$ represents the usual standing wave solution for a span with undamped vibration. This represents the trivial case in which the inner and outer cables are vibrating in such exact synchronism that they do not react with one another. Since any force tending to cause the system to oscillate necessarily acts on the exterior conductor, asymmetry is introduced from the beginning of the motion, and synchronism cannot exist.

The second summation represents the actual physical case when the 2 cables react one against the other, but it will be noticed that the whole disturbance dies out because of the factor $\epsilon^{-R t / 2 m}$. If the damping coefficient $R$ of the packing material is large, the quantity $\alpha_{n}$ is imaginary for small values of $n$, showing that in such a case vibrations having great loop lengths do not occur. In any case the exponential damping term soon nullifies the entire motion.

\section{Distinction Between}

\section{Distributed and Lumped Systems}

It is evident that there is a difference between coupled systems having lumped constants and systems having distributed constants. In lumped systems the coupling causes the natural frequency of the resulting system to be removed from that of the individual systems, but in distributed systems both uncoupled systems have an infinite number of natural frequencies, and so does the coupled system. It does not seem possible, therefore, by coupling 2 cables to remove the natural frequency of the system beyond the range of the most prevalent objectionable frequency of the system produced by wind eddies.

The chief advantage resulting from the type of system here considered is the fact that a material having a large damping coefficient $R$ may be employed; hence, vibrations of a transient character are reduced promptly.

\section{TRAVELING WAVES}

Before analyzing the forced vibration it is of interest to study the behavior of a traveling wave on the system under consideration. If a traveling wave of angular frequency $\omega$ and velocity $v$ is considered, the following solutions are applicable:

1. Inner and Outer Cables in Exact Synchronism. This represents the trivial case in which synchronism between inner and outer conductors is so perfect that there is no interaction.

$u_{2}=A \cos \omega(t-s / v)+B \cos \omega(t+s / v)$

2. Loose Coupling or High Frequencies. If the spring effect of the packing is not excessive, it may be assumed that $k / \omega^{2} m$ is much less than unity. The solution is:

$$
\begin{array}{r}
u_{2}=A \epsilon \frac{R \omega s}{\overline{2 m a \sqrt{\omega^{2}-k / m}}} \cos \omega\left[t+\frac{s \sqrt{\omega^{2}-k / m}}{\omega a}\right]+\cdot \\
B \epsilon-\frac{R \omega s}{2 m a \sqrt{\omega^{2}-k / m}} \cos \omega\left[t-\frac{s \sqrt{\omega^{2}-k / m}}{\omega a}\right]
\end{array}
$$

This equation indicates damped traveling waves whose damping and velocity of propagation depend upon the frequency; hence, a complex wave would suffer distortion.

3. Frequency $\omega$ Approximately Equal to $\sqrt{k / m}$. In this case the waves are of the form

$u_{2}=A \epsilon^{-\alpha_{1} s} \cos \omega\left[t-\beta_{1} S\right]+$

$B_{\epsilon}{ }^{\alpha_{1} s} \cos \omega\left[t+\beta_{1} s\right]$

where

$\alpha_{1}=\frac{1}{a \sqrt{2}} \sqrt{\frac{R \omega}{m}}$ and $\beta_{1}=\frac{1}{a \sqrt{2}} \sqrt{\frac{R}{\omega m}}$

and distortion is produced as before.

4. Low Frequencies or Close Coupling. This case, which may be approximated closely in practice, represents waves traveling with a velocity $v=$ $2 a \sqrt{\mathrm{km} / R}$ which is great, since in general $R$ is small. The attenuation in this case is extremely great, and there is no distortion.

$u_{2}=A \epsilon^{-\alpha s} \cos \omega[t-\beta s]+$

$B \epsilon^{+\alpha s} \cos \omega[t+\beta s]$

in which $\omega^{2}<k / m, \alpha=(1 / a) \sqrt{k / m}$ and

$\beta=R / 2 m a \sqrt{k / m}$.

\section{FORCED VIBRATION}

If a force of the form $A_{2} \cos \omega t$ is assumed to be of constant amplitude throughout the length of the conductor and of an oscillatory character acting at
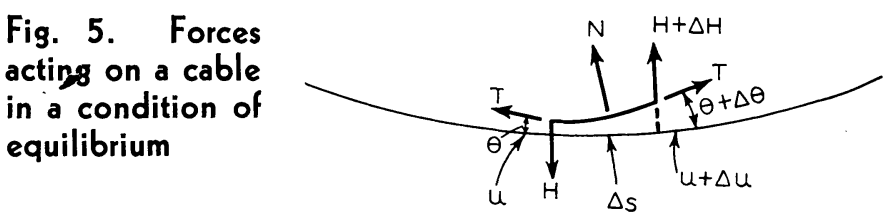

every point of the outer cable, the following equation is obtained for the vibration of the outer cable in the steady state:

$$
u_{2}=\frac{A_{2}}{\omega^{2}} \sqrt{\frac{\left(k-\omega^{2} m_{1}\right)+R^{2} \omega^{2}}{\left[\omega^{2} m_{1}-\left(1+\frac{m_{1}}{m_{2}}\right)^{2}\right]^{2}+R^{2} \omega^{2}\left(1+\frac{m_{1}}{m_{2}}\right)^{2}}}
$$

where

$$
\theta_{1}-\theta_{2}=\theta_{3} \quad \theta_{1}=\tan ^{-1} \frac{R / m_{1}}{k / m_{1}-\omega^{2}} \quad \theta_{2}=\frac{\omega \delta R}{\omega^{2}-\delta k} \quad \delta=\frac{1}{m}
$$

The transient solution must be added to equation 28 in order to satisfy the boundary values. This complex expression gives an approximate indication of the behavior of the system under the action of a hypothetical wind.

It will be noticed that in every case it is the factor $R$ that is active in reducing the vibration. This is only a special application of the principle that energy must be absorbed in order to damp vibra- 
tion. The energy absorption in the case of Preiswerk's loose core cables must come from the shock of bumping, and, of course, this action must be destructive. In the above case no such destructive action exists.

\section{Appendix}

\section{Derivation OF Equations}

The equation of the catenary curve, which is the static equilibrium position of the cable, is:

$y=\frac{H}{w} \cosh \frac{w w}{H}$

As a typical case for a cable having a diameter of one inch and a span length of 1,200 feet, let

$$
\begin{aligned}
H & =\text { the horizontal component of } T \\
T & =\text { tension } \\
w & =\text { weight per unit length } \\
l & =\text { span length }
\end{aligned}
$$

but

$H=8,500$ pounds $=T$ approximately

$w=0.858$ pounds per foot

then

$\frac{w}{H}=\frac{0.858}{8,500}=10^{-4}$ approximately

and the equation of the span can be taken to be approximately that of a parabola

$y=w x^{2} / 2 H$

then 1

$\frac{d y}{d x}=\frac{w x}{H}$ and $\frac{d^{2} y}{d x^{2}}=\frac{w}{H}$

The curvature of this span is given by

$K=\frac{1}{R}=\frac{d^{2} y / d x^{2}}{\left[1+\left(\frac{d y}{d x}\right)^{2}\right]^{3 / 2}}=\frac{w}{T}$

approximately, since for all points $\left(w x / H^{2}\right)$ may be neglected in comparison to unity. Thus it is seen that the curvature of the static curve may be regarded as having the small constant value $w / H$, or the radius of curvature $R$ as the large constant value $T / w$ for a typical span.

The fact that the equilibrium curve can be considered with very little error as a circle of large radius of curvature $T / w$ simplifies the analysis greatly.

Let $s$ be the position of static equilibrium of the cable and measure $u$ in the normal direction to the static position of equilibrium $s$. Now consider the shearing force $H$ at the point $s$ (figure 5) and the shearing force $H+\Delta H$ at the point $S+\Delta S$.

Since small deviations are being considered from the static position of equilibrium, which takes into account the weight of the cable, the weight of the cable may be neglected and components of shearing force and tension may be taken in the direction $u$ normal to the cable at the point $s$. Consider, in addition, an outside force $N$ per unit length acting normal to the cable.

Equating forces in the $u$ direction,

$T \sin \theta+H \cos \theta=T \sin (\theta+\Delta \theta)+$

$$
(H+\Delta H) \cos (\theta+\Delta \theta)+N d S
$$

$\lim _{\Delta \theta \rightarrow 0} \sin (\theta+\Delta \theta)=\sin \theta+\frac{\partial^{2} u}{\partial s^{2}} d S$

$\lim _{\Delta \theta \rightarrow 0} \cos (\theta+\Delta \theta)=\cos \theta$ then

$T \frac{\partial^{2} u}{\partial s^{2}} d S+\Delta H+N d S=0$

$\Delta H=\frac{\partial H}{\partial s} d S$

and

$T \frac{\partial^{2} u}{\partial s^{2}}+\frac{\partial H}{\partial s}=-N$

Since only transverse vibrations are of importance here, the components of force along $s$ need not be written.

There is another condition expressing the fact that there is no

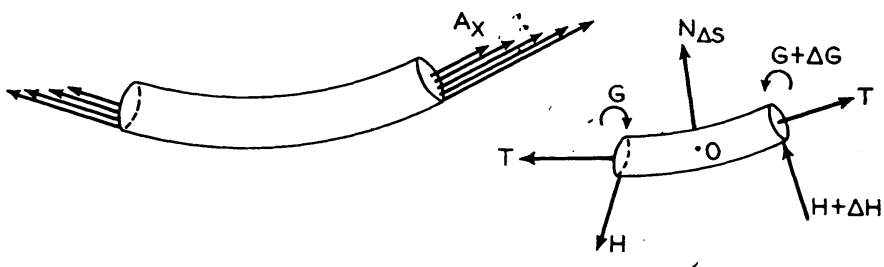

Fig. 6. Free body diagram of a differential length of a conductor vibrating transversely

tendency to turn the element about an axis parallel to the $z$-axis.

Suppose that the moment of all the normal forces $A_{x}$ on one end of a differential element is $-G$ and at the other end is $G+$ $\frac{\partial G}{\partial s} d s$ as indicated in figure 6 .

Taking moments about $o$

$G=G+\Delta G+H \frac{\Delta S}{2}+(H+\Delta H) \frac{\Delta S}{2}$

then

$-\Delta G=H \Delta S+\frac{(\Delta H)(\Delta S)}{2}$

$\lim _{\Delta s \rightarrow 0} H=-\frac{\partial G}{\partial s}$ and $\frac{\partial H}{\partial s}=-\frac{\partial^{2} G}{\partial s^{2}}$

The moment $G$ may be considered as a result of the stresses of the longitudinal fibers of the cable in bending. Consider a bent section of wire so that it has a radius of curvature $R$ and let $q$ be the distance of a given fiber from the neutral surface. Since the neutral surface is the surface at which the fibers are in a state of equilibrium,

$\frac{\text { stretched length }}{\text { unstretched length }}=\frac{R-q}{R}=1-\frac{q}{R}$

Let $E$ be Young's modulus of elasticity for the material of the wire, then

normal stress $=A_{x}=-\frac{E q}{R}$

The moment about the elastic surface $o-o$ (figure 7 ) is:

$G=-\int_{S} \int A_{x} q d S=\frac{E}{R} \int_{S} \int q^{2} d S$

but $\int_{s} \int g^{2} d s$ is the moment of inertia $I$ of the plane area of the normal cross-sectional area of the conductor with respect to the diameter $0-0$.

It has been found that for a typical static span the curvature is very small, being of the order $w / T$; hence, any appreciable curvature must be due to the dynamic wave. For the radius of curvature of the dynamic waves occurring on a tightly stretched transmission line $1 / R=\partial^{2} u / \partial S^{2}$ and the unbalanced force $N$ is defined by 
d'Alembert's principle $-N=m \partial^{2} u / \partial t^{2}-F$ where $m$ is the mass per unit length, $\partial^{2} u / \partial t^{2}$ is the acceleration, and $F$ is any other force per unit length.

The value of $N$ is

$-N=T \frac{\partial^{2} u}{\partial s^{2}}+\frac{\partial H}{\partial s}$ where $\frac{\partial H}{\partial s}=-\frac{\partial^{2} G}{\partial s^{2}}$ and $G=E I \frac{\partial^{2} u}{\partial s^{2}}$

and the equation of motion becomes

$m \frac{\partial^{2} u}{\partial t^{2}}+E I \frac{\partial^{4} u}{\partial s^{4}}=T \frac{\partial^{2} u}{\partial s^{2}}+F(s, t)$

The EI term involves the effect of rigidity. Monroe and Templin ${ }^{7}$ have made some measurements of $E I$ for a cable subjected to tension.

Traveling Waves

Placing $F=0$, assuming $u=\cos \omega(t-s / v)$ and substituting in

$m \frac{\partial^{2} u}{\partial t^{2}}+E I \frac{\partial^{4} u}{\partial s^{4}}=T \frac{\partial^{2} u}{\partial s^{2}}$

where

$k=1 / v \quad T / m=a^{2}$ and $E I / m=\beta^{2}$

produces the following result:

$2 k^{2}=-\frac{a^{2}}{\beta^{2} \omega^{2}} \pm \sqrt{\frac{a^{4}}{\beta^{4} \omega^{4}}+\frac{4}{\beta^{2} \omega^{2}}}$

Expanding, $k .= \pm 1 / a$ or $v= \pm a \sqrt{T / m}$ provided that $x^{2}$ is negligible in comparison with $x$ when $f=$ frequency, $\omega=2 \pi f$ and

$x=\frac{4 E I m \omega^{2}}{T^{2}}$

If $m=\pi \rho r^{2}$ and $\bar{I}=\pi r^{4} / 4$ in the above expression,

$x=\frac{4 \rho \pi E r^{8} f^{8}}{T^{2}}$

The units used are the foot and the pound. $E$ must be expressed in pounds per square foot and $I$ in (feet).

In the general case in which $x$ is not small, the above equation may be solved for $k$, and since $k=1 / v$,

$0=\omega \sqrt{\frac{2 E I}{T}} \sqrt{\frac{1}{\sqrt{1+\frac{4 E I m \omega^{2}}{T^{2}}}-1}}$

\section{Standing Waves on Rigid Conductors}

Equation 1a may be solved by the usual method of separation of variables with the assumption that $u=\phi(S) \psi(t)$. Making use of the boundary conditions $u=0$ at $s=0$ and $u=0$ at $s=l$ leads to an extremely complicated transcendental equation for $n$, as follows:

$\frac{\sinh r_{1} l \sin r_{2} l}{1-\cosh r_{1} l \cos r_{2} l}+\frac{2 n \beta}{a^{2}}=0$

where

$r_{1}^{2}=\frac{a^{2}}{2 \beta^{2}}+\frac{1}{2} \sqrt{\frac{a^{4}}{\beta^{4}}+\frac{4 n^{2}}{\beta^{2}}} \quad r_{2}^{2}=-\left(\frac{a^{2}}{2 \beta^{2}}-\frac{1}{2} \sqrt{\frac{a^{4}}{\beta^{4}}+\frac{4 n^{2}}{\beta^{2}}}\right)$

$\beta^{2}=\frac{E I}{m} \quad f=\frac{n}{2 \pi}$

It is found for the usual constants of a transmission line that $r_{1}$ is large and $r_{2}$ is small; therefore, the approximations cosh $r_{1} l=\sinh$ $r_{1} l$ and $\cos r_{2} l \rightarrow 1$ are justifiable.

The above expression then reduces to

$\tan r_{2} l=\frac{2 n \beta}{a^{2}}$ where $r_{2}^{2}=\frac{n^{2}}{a^{2}}$ and is subject to the very accurate approximate solution

$n=\frac{r \pi}{l} \sqrt{\frac{T}{m}}\left(1+\frac{2}{l} \sqrt{\frac{E I}{T}}\right)$

or

$f=\frac{n}{2 \pi}=\frac{r}{2 l} \sqrt{\frac{T}{m}}\left(1+\frac{2}{l} \sqrt{\frac{E I}{T}}\right)$

in which $r=0,1,2, \ldots$

\section{DAMPED VibRations}

In this case the important results are the values of $v$ in the traveling wave solution $u=A \cos \omega(t-s / v)$. By substituting these values in the equation $m \partial^{2} u / \partial t^{2}=T \partial^{2} u / \partial s^{2}-R \partial u / \partial t$, making use of the exponential form for the trigonometric relations, and assuming that the damping factor $R$ is small, it is found that

$u=A \epsilon^{-\frac{R s}{2} \sqrt{\frac{1}{m T}} \cos \omega(t-s / a)}$

Standing Waves With Damping

An approximate general solution of the equation $m \partial^{2} w / \partial t^{2}=$ $T \partial^{2} u / \partial s^{\prime}-R \partial u / \partial t$ may be obtained by making the substitution $u=y(s, t) \epsilon-\frac{R t}{2 m}$ which produces the expression

$\frac{\partial^{2} y}{\partial t^{2}}-\frac{R^{2}}{4 m^{2}} y=\frac{T}{m} \frac{\partial^{2} y}{\partial s^{2}}$

Since $R / m$ is small, however, this term may be neglected on the supposition that $y$ is small. Then

$\frac{\partial^{2} y}{\partial t^{2}}=\frac{T}{m} \frac{\partial^{2} y}{\partial s^{2}}$

whose solution is:

$y=\phi_{1}(s-a t)+\phi_{2}(s+a t)$

Hence

$u=\epsilon^{-\frac{R t}{2 m}}\left[\phi_{1}(s-a t)+\phi_{2}(s+a t)\right]$

StaNDING Waves

The solution for standing waves with damping may be obtained easily by the method of separation of variables. The boundary

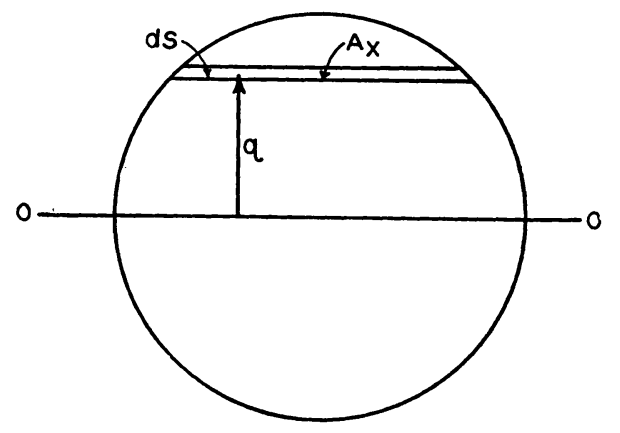

Fig. 7. Normal cross section of a vibrating conduc. tor, showing the quantities involved in the derivation of the dynamic equa-

conditions of fixity at the extremities must be imposed, and with the condition that $R$ is small

$u=\epsilon^{-\frac{R t}{2 m}} \sum_{1}^{\infty}\left(A_{n} \sin \beta_{n} t+C_{n} \cos \beta_{n} t\right) \sin \frac{n \pi s}{l}$

$\beta_{n}=\frac{1}{2} \sqrt{\frac{4 n^{2} \pi^{2} a^{2}}{l^{2}}-\frac{R^{2}}{m^{2}}}$ 
Forced Oscillations

Consider the equation

$m \frac{\partial^{2} u}{\partial t^{2}}+R \frac{\partial u}{\partial t}=T \frac{\partial^{2} u}{\partial s^{2}}+M_{T} D V_{W}^{2} \cos \omega t-M_{D} D V_{W} \frac{\partial u}{\partial t}$

Let

$p=\frac{1}{m}\left[R+M_{D} D V_{W}\right] a^{2}=T / m$ and $A=\frac{M_{T}}{m} D V_{W}{ }^{2}$

then

$\frac{\partial^{2} u}{\partial t^{2}}+p \frac{\partial u}{\partial t}=a^{2} \frac{\partial^{2} u}{\partial s^{2}}+A \cos \omega t$

Assuming $u=v(s)^{j \omega t}$ and retaining only the real part of the solution,

$\frac{\partial^{2} u}{\partial t^{2}}+p \frac{\partial u}{\partial t}=a^{2} \frac{\partial^{2} u}{\partial s^{2}}+A \epsilon \epsilon^{j \omega t}$

if

$K^{2}=\frac{1}{a^{2}}\left[\omega^{2}-j \omega p\right]$

then

$\frac{d^{2} v}{d S^{2}}+K^{2} v=-\frac{A}{a^{2}}$

or

$v=C_{1} \cos K s+C_{2} \sin K s-\frac{A}{a^{2} K^{2}}$

The boundary conditions are: 1. At $s=0, v=0$. 2. At $s=l$, $v=0$

Then<smiles>CCCC</smiles>

$v=\frac{A}{a^{2} K^{2} \sin K l}[\sin K s+\sin K(l-s)-\sin K l]$

where

$K^{2}=\frac{\omega}{a^{2}}[\omega-j p]$

It is required that

$[\omega-j p]^{1 / 2}=\omega^{1 / 2}\left[1-1 / 2 j p / \omega+1 / s p^{2} / \omega^{2} \ldots\right]$

Since in general

$p<\omega$

$[\omega-j p]^{1 / 2}=\omega^{1 / 2}[1-1 / 2 j p / \omega]$

and

$K=\frac{\omega}{a}[1-1 / 2 j p / \omega]$

for practical purposes. However

$\sin (x-j y)=\sin x \cosh y-j \cos x \sinh y$

therefore

$\sin K l=\sin \left[\frac{l}{a}\left(\omega-j \frac{p}{2}\right)\right]=\sin \frac{\omega l}{a} \cosh \frac{p l}{2 a}-j \cos \frac{\omega l}{a} \sinh \frac{p l}{2 a}$

At resonance frequency $\sin (\omega l / a)=0 \quad(\omega l / a)=n \pi \quad n=0,1, \ldots$ and $f=(n / 2 l) \sqrt{T / m}$

In addition, $\cos (\omega l / a)= \pm 1$ depending upon whether $n$ is odd or even. Then $|\sin K l|_{\text {resonance }}=\sinh \frac{p l}{2 a}=\sinh \left\{\frac{l}{2} \sqrt{\frac{m}{T}}\left(R+M_{D} D V_{W}\right)\right\}$

The imaginary part of the other terms is of no interest, and may be neglected in the solution

$u=\frac{A}{a^{2} K^{2} \sin K l}[\sin K s+\sin K(l-s)-\sin K l] \cos \omega t$

It will be noticed that $\sin K l$ never can equal zero and that its imaginary part may be neglected except in the discussion of resonance; hence,

$K=\frac{\omega}{a}$ and $|\sin K l|_{\text {resonance }}=\sinh \frac{p l}{2 a}$

The phase angle which should be introduced in the cos $\omega t$ term also may be discarded as having no bearing on the discussion, since $\omega>p$ in the practical case.

Hence

$u=\frac{23.8 M_{T} D^{3}}{w \sin K l}\left[\sin K_{s}+\sin K(l-s)-\sin K l\right] \cos \omega t$

by obvious transformations and changes in units.

\section{ENERgy CoNSIDERations}

Genieral expressions for the energy of transverse vibrations in an oscillating span may be obtained easily for the usual case in which the tension is great in comparison to the mass per unit length. The kinetic energy per unit length is seen to be $1 /{ }_{2} m(\partial u / \partial t)^{2}$ from general principles.

The potential energy equation requires a little more care. Let $s$ be the length of the cable in the normal catenary form as the position of equilibrium and $s_{1}$ be the dynamic length of the cable.

Since the weight per unit length is small compared to the tension, the potential energy is the work necessary to stretch against the tension because of the loops formed by the oscillation. Potential energy stored in bending is neglected.

The potential energy $V$ per unit length is:

$V=T\left(\frac{\partial s_{1}}{\partial s}-1\right)$ but $d s_{1}=\sqrt{d s^{2}+d u^{2}} \frac{\partial s_{1}}{\partial s}=\sqrt{1+\left(\frac{\partial u}{\partial s}\right)^{2}}$

and

$\frac{\partial s_{1}}{\partial s}=\left[1+\left(\frac{\partial u}{\partial s}\right)^{2}\right]^{1 / 2}=1+\frac{1}{2}\left(\frac{\partial u}{\partial s}\right)^{2}+\ldots+$

then

$V=\frac{1}{2}\left(\frac{\partial u}{\partial s}\right)^{2} T$

$\partial u / \partial s$ is small but not zero and lower order quantities have been neglected.

Assume now a traveling wave of the form $u=A \cos (\omega t+\beta s)$ where $\beta$ and $\omega$ must be such as to satisfy $m \partial^{2} u / \partial t^{2}=T \partial^{2} u / \partial s^{2}$. By carrying out the above differentiations and averaging throughout a cycle it is found that average energy per unit length

$W=1 / 2 m \omega^{2} A^{2}$.

\section{ANALYSIS OF VibRation DAMPER}

Let the damper be represented schematically as in figure 8 , and let

$x_{F}=$ absolute displacement of the damper frame relative to a point fixed in space

$x_{M}=$ displacement of the mass $M$ from the point of equilibrium relative to the frame

$x_{A}=$ absolute displacement of the mass $M$ relative to a fixed point in space

$\mu \quad=$ combined spring constant of both springs

$R=$ damping coefficient of the springs

The equations of motion are:

$M \frac{d^{2} x_{A}}{d t^{2}}+R \frac{d x_{M}}{d t}+\mu x_{M}=0$ 
and

$$
x_{A}=x_{M}+x_{F}
$$

Assume that the cable is vibrating in such a manner that at the point at which the damper is attached it is performing an oscillation of the type $x_{F}=D \cos \omega t$

Then the equation of motion is

$\frac{d^{2} x_{M}}{d t^{2}}+\frac{R}{M} \frac{d x_{M}}{d t}+\frac{\mu}{M} x_{M}=\omega^{2} D \cos \omega t$

which has the steady state solution

$x_{. Y}=\frac{M \omega D \sin (\omega t-\phi)}{\sqrt{R^{2}+(\omega M-\mu / \omega)^{2}}}$ and $\tan \phi=\frac{\omega M-\mu / \omega}{R}$

\section{Power Consumed}

Let $W$ be the amount of energy dissipated in the damper. From fundamentals $d W=F d S$. Now at ime $t$

$d S=d x_{M}$ and $F=R \frac{d x_{M}}{d t}$

Let

$M D \omega=E_{0}$

$Z=\sqrt{R^{2}+(\omega M-\mu / \omega)^{2}}$

$R \frac{d x_{M}}{d t}=\frac{R E_{0} \omega \cos (\omega t-\phi)}{Z}$

then

$d W=\frac{R E_{0}^{2} \omega^{2} \cos ^{2}(\omega t-\phi) d t}{Z^{2}}$

Since the average power dissipated is of importance, this expression must be integrated through a complete cycle and divided by the elapsed time of the cycle. The result of these operations is:

$P_{\text {average }}=\frac{R M^{2} D^{2} \omega^{4}}{2\left[R^{2}+\left(\omega M-\frac{M}{\omega}\right)^{2}\right]}$

The various expressions given by equations 15,16 , and 17 for the optimum values of the constants may be obtained by maximizing equation 14 .

\section{REACTION OF DAMPER ON CONDUCTOR}

The reaction of the moving mass of the damper on its frame, which is equivalent to its reaction on the conductor, if the mass of the frame be neglected, is defined as

$F=\mu x_{M}+R \frac{d x_{M}}{d t}=\frac{\omega M D \sqrt{\mu^{2}+R^{2} \omega^{2}}}{\sqrt{R^{2}+(\omega M-\mu / \omega)^{2}}} \cos (\omega t-\dot{\phi}-\beta)$

where

$\phi=\tan ^{-1} \frac{\omega M-\mu / \omega}{R}$ and $\beta=\tan ^{-1} \mu / \omega R$

by obvious transformations

\section{Extremities Subject to Yielding}

The vertical component of tension at the support (figure 9 ) is

$-T \sin (\theta+\alpha)=-T(\sin \theta \cos \alpha+\cos \theta \sin \alpha)$

but $\alpha$ is small; therefore $\cos \alpha \rightarrow 1, \sin \alpha \rightarrow 0$ and the vertical component of tension is

$-T\left[\sin \theta+\cos \theta\left(\frac{\partial u}{\partial s}\right)_{s=0}\right]$
The first term of this expression represents the downward pull due to the weight of the cable and the second term represents fluctuations in force due to the dynamic oscillation of the cable. For the usual cable span the angle $\theta$ is quite small, as may be seen from the equations of the catenaries in which these spans lie; therefore, the approximation $\cos \theta=1$ is valid.

The boundary equations at the extremities are:

$M \frac{\partial^{2} u}{\partial t^{2}}+\mu u=T \frac{\partial u}{\partial s} \quad$ at $s=o$

$M \frac{\partial^{2} u}{\partial t^{2}}+\mu u=-T \frac{\partial u}{\partial s}$ at $s=l$

where the supports are constrained to move in a vertical line and $M$ and $\mu$ are the mass and the spring constant of the support, respectively. The wave equation $m \partial^{2} u / \partial t^{2}=T \partial^{2} u / \partial s^{2}$ must be solved subject to these boundary conditions. By the usual method of separation of variables, the solution is

$u=(A \sin r s+D \cos r s) \sin r a t$

where $A$ is arbitrary, but is connected to $D$ by the relation

$\frac{A}{D}=\frac{\mu-M r^{2} a^{2}}{r T}$

and the possible values of $r$ are given by the complicated transcendental equation

$\tan r l=\tan 2 \phi$

where

$\phi=\tan ^{-1} \frac{\mu-M a^{2} r^{2}}{r T}$

Having determined $r$, use may be made of the principle of superposition, which always applies to linear equations, and a particular solution capable of expressing any arbitrary initial configuration will result. The case of slight immobility has been discussed previously.

Reflection of Traveling Waves From Movable Supports

Consider 2 adjacent spans connected by a movable support that has some degree of freedom in a vertical line. Let this support have a mass $M$ which is constrained to move in a vertical line by a spring having a spring constant $\mu$ and a damping coefficient $R$.

Let

$T_{1}=$ the tension in span 1

$T_{2}=$ the tension in span 2

$m_{1}=$ the mass per unit length of span 1

$m_{2}=$ the mass per unit length of span 2

$H_{1}=$ the shearing force exerted on the support as a consequence of rigidity of cable 1

$H_{2}=$ a similar quantity for cable 2

$\theta_{1}=$ angle with the horizontal made by the catenary of cable 1 at the point of support

$\theta_{2}=$ similar quantity for cable 2
Fig. 8. Diagram of one type of vibration damper
POINT FIXED IN SPACE

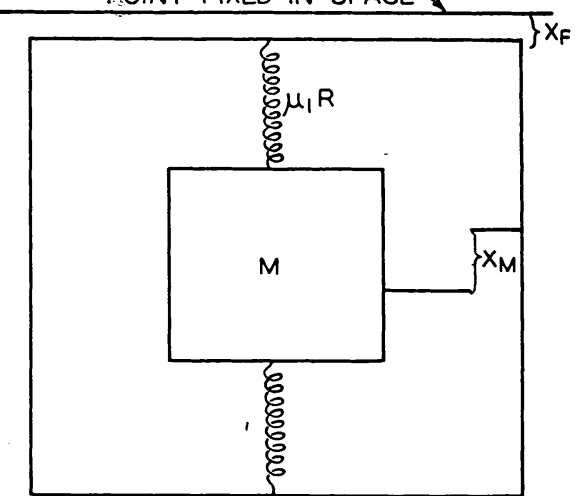


Note that the angles $\theta_{1}$ and $\theta_{2}$ are given respectively by

$\theta_{1}^{\prime}=\tan ^{-1}\left[\sinh \frac{l_{1} w_{1}}{T_{1}}\right]$

$\theta_{2}=\tan ^{-1}\left[\sinh \frac{l_{2} w_{2}}{T_{2}}\right]$

where $l_{1}$ and $l_{2}$ and $w_{1}$ and $w_{2}$ are the respective lengths and weights per unit length of the 2 cables. In general, it may be seen that these angles are quite small.

The vertical component of force exerted by the cables (figure 10) on the support is

$$
\begin{aligned}
& F_{y}=T_{2} \sin \left(\frac{\partial u_{2}}{\partial s_{2}}-\theta_{2}\right)+H_{2} \sin \left(\frac{\pi}{2}-\theta_{2}\right)+ \\
& T_{1} \sin \left(\frac{\partial u_{1}}{\partial s_{1}}-\phi_{1}\right)+H_{1} \sin \left(\frac{\pi}{2}-\phi_{1}\right)
\end{aligned}
$$

where $u_{1}$ and $u_{2}$ are measured at right angles to the static position of equilibrium.

The boundary conditions are:

$u_{1} \sin \left(\frac{\pi}{2}-\theta_{1}\right)=u_{2} \sin \left(\frac{\pi}{2}-\theta_{2}\right)$

and

$M \frac{\partial^{2} u_{v}}{\partial t^{2}}+R \frac{\partial u_{v}}{\partial t}+\mu u_{v}=F_{y}$

Let

$u_{v}=u_{2} \cos \theta_{2}=u_{I} \cos \theta_{1}$ and $u_{1}=u_{I}+u_{R}$

where

$u_{I}=$ incident wave traveling on span number 1

$u_{R}=$ reflected wave traveling on span number 1

$u_{2}=$ the refracted wave on span number 2

Then

$u_{I}=A \cos \omega\left(t+s_{1} / v_{1}\right)=$ real past of $A \epsilon^{j \omega\left(t+s_{1} / v_{1}\right)}$

$u_{R}=B \cos \omega\left(t-s_{1} / v_{1}\right)=$ real part of $B \epsilon^{j \omega t}\left(t-s_{1} / v_{1}\right)$

$u_{2}=C \cos \omega\left(t-s_{2} / v_{2}\right)=$ real part of $C \epsilon^{j \omega\left(t-s_{2} / v_{2}\right)}$

Substituting in the boundary conditions,

$F_{y}=C \epsilon^{j \omega\left(t-s_{2} / v_{2}\right)}\left[-\omega^{2} M+R j \omega+\mu\right]$

Making use of the expression for the shearing force $H$,

$H=-\frac{\partial G}{\partial S}=-E I \frac{\partial^{3} u}{\partial s^{3}}$

Let

$\sin \frac{\partial u_{2}}{\partial s_{2}} \longrightarrow \frac{\partial u_{2}}{\partial s_{2}}$ and $\cos \frac{\partial u_{2}}{\partial s_{2}} \longrightarrow 1$

since oscillations of small amplitude are being considered. The expression for the vertical components of forces becomes

$F_{y}=\cos \theta_{2}\left(T_{2} \frac{\partial u_{2}}{\partial S_{2}}-E_{2} I_{2} \frac{\partial^{3} u_{2}}{\partial s_{2}{ }^{3}}\right)+$

$\cos \theta_{1}\left(T_{1} \frac{\partial u_{1}}{\partial s_{1}}-E_{1} I_{1} \frac{\partial^{3} u_{1}}{\partial s_{1}^{3}}\right)-\left(W_{1}+W_{2}\right)$

where $W_{1}$ and $W_{2}$ represent $1 / 2$ the weights of the cables of span number 1 and span number 2 , respectively. These quantities represent a static load on the spring assumed at the supports, and do not influence the dynamic condition.

Substituting the assumed wave solutions of the differential equations of motion, and equating the above 2 expressions for $F_{y}$, it is found that

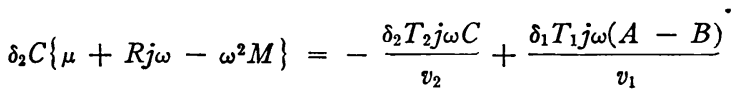

$\delta_{1}(A+B)=\delta_{2} C, \delta_{2}=\cos \theta_{2}$ and $\delta_{1}=\cos \theta_{1}$

in which the effect of the shearing force $H$ has been neglected, because

$H_{2}=-E I \frac{\partial^{3} u_{2}}{\partial s_{2}{ }^{3}}=-E I \frac{j \omega^{3}}{v_{2}{ }^{3}} C \epsilon^{j \omega\left(t-s_{2} / v_{2}\right)}$

It has been indicated previously that $v_{2}=\sqrt{T_{2} / m_{2}}$ and for the usual size of cable and frequency of vibration this term may be neglected with respect to the term, $T_{2} \partial u_{2} / \partial s_{2}$ provided that $\omega<v_{2}$ and $E I<T$.

Solving the 2 equations above for $C$ and $B$ in terms of $A$ and retaining only the real part of the solutions results in equations 20 , $20 a$, and $20 b$.

\section{REFLECTION FROM DAMPER}

The calculation of the reflection from the damper proceeds in the same manner as above. As a boundary condition the force of re-
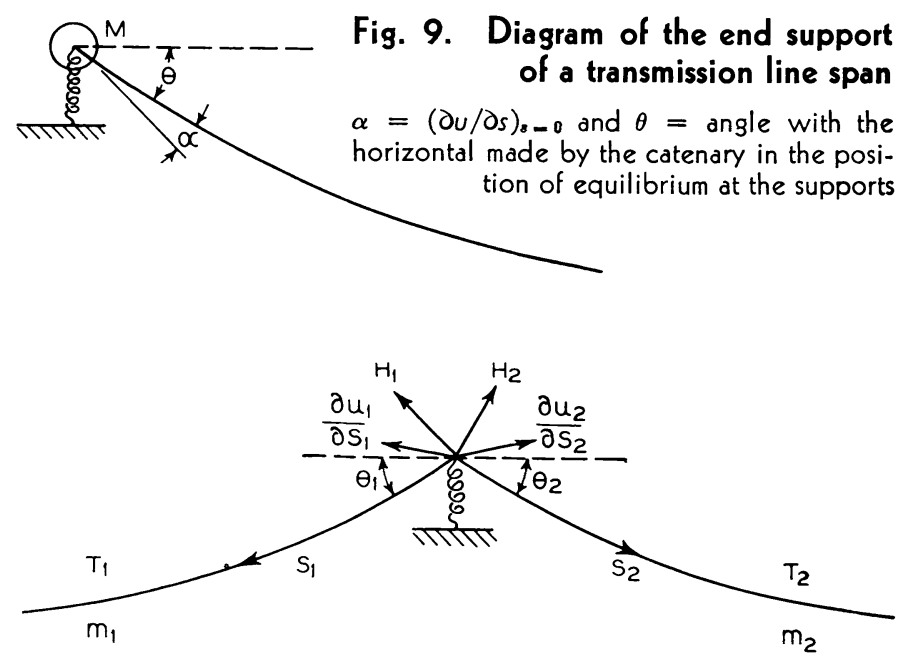

Fig. 10. Force diagram of adjacent spans connected by a movable support

action of the damper as calculated in equation 18 (converted to the exponential form) must be equated as follows:

$\frac{\omega M C \sqrt{\mu^{2}+R^{2} \omega^{2}}}{\sqrt{R^{2}+(\omega M-\mu / \omega)^{2}}} \epsilon^{j \omega t} \epsilon^{j \theta}=T\left(\frac{\partial u_{T}}{\partial s}\right)-T\left(\frac{\partial u_{I}}{\partial s}+\frac{\partial u_{R}}{\partial s}\right)$.

where

$\theta=-(\phi+\beta)$ and $u_{T}=u_{I}+u_{R}$

Equation 21 is produced by substituting the assumed expressions for the traveling waves, satisfying the boundary equations, retaining only the real terms, and making certain trigonometric and algebraic reductions.

Hollow Cables

The analysis of coupled hollow cables is merely a solution of the simultaneous equations

$p^{2} u_{1}=a^{2} \sigma^{2} u_{1}+K_{1}\left(u_{2}-u_{1}\right)+E_{1} p\left(u_{2}-u_{1}\right)$

$p^{2} u_{2}=a^{2} \sigma^{2} u_{2}+K_{2}\left(u_{1}-u_{2}\right)+E_{2} p\left(u_{1}-u_{2}\right)+$

$A_{2} \cos (\omega t+\beta s)$

where

$p=\frac{\partial}{\partial t} \quad \sigma=\frac{\partial}{\partial s} \quad$ and $\quad a^{2}=\frac{T_{1}}{m_{1}}=\frac{T_{2}}{m_{2}}$

If $u_{1}$ is eliminated from these 2 equations, and only the real parts 
are retained, the solution is:

$\left[\alpha^{2}+(s+1) \alpha \delta\right] \iota_{2}=A_{2}\left(-\omega^{2}+a^{2} \beta^{2}+K_{1}+E j \omega\right) \epsilon^{j(\omega t+\beta s)}$

where the operators are defined as

$\alpha=p^{2}-a^{2} \sigma^{2} \quad \delta=K_{1}+E_{1} p$ and $s=m_{1} / m_{2}$

Free Vibrations, Standing Waves

In this case $A_{2}$ is zero and the expression

$\left[\alpha^{2}+(s+1) \alpha \delta\right] u_{2}=0$

must be solved by the assumption that

$u_{2}=C_{\epsilon}^{j(\omega t+\beta s)}$

The boundary condition of fixity at the extremities produces the result

$\boldsymbol{\beta}=n \pi / l$ where $n=0,1,2, \ldots$

Equation 28 connects $\omega$ with $\beta$. Using the principle of superposition because of the linearity of the equation

$u_{2}=\sum_{0}^{\infty}\left(A_{n} \sin \frac{\pi a n t}{l}+B_{n} \cos \frac{\pi a n t}{l}\right) \sin \frac{\pi n s}{l}+$

$\epsilon^{-\frac{R}{2 m} t} \sum_{0}^{\infty}\left(C_{n} \sin \alpha_{n} t+D_{n} \cos \alpha_{n} t\right) \sin \frac{n \pi s}{l}$

where

$\frac{1}{m}=\frac{1}{m_{1}}+\frac{1}{m_{2}}$ and $\alpha_{n}=\sqrt{\frac{k}{m}+\frac{a^{2} n^{2} \pi^{2}}{l^{2}}-\frac{R^{2}}{4 m^{2}}}$

Traveling Waves

Substitute the expression $u_{2}=A e^{j \omega(l-s / v)}$ representing a traveling wave, in the equation $\left[\alpha^{2}+(s+1) \alpha \delta\right] u_{2}=0$. This substitution produces the expressions

$v= \pm a$ and $\frac{a^{2}}{v^{2}}=1-\frac{1}{\omega^{2} m}\left(k^{\prime}+R j \omega\right)$

Solving for $v$ in the second expression and substituting the result in the above assumed solution, introduces the various expressions of equations $24,25,26$, and 27 with the approximations that are mentioned in connection with them.

ForCed Vibrations

In the case of forced vibrations $A_{2}$ is not zero and the equation

$\left[\alpha^{2}+(s+1) \alpha \delta\right] u_{2}=A_{2}\left(-\omega^{2}+a^{2} \beta^{2}+K_{1}+E_{1} r \omega\right) e^{j(\omega t+\rho s)}$

must be solved. This is easily done by assuming

$u_{2}=C_{\epsilon}{ }^{j(\omega t+\beta s)}$

and substituting and solving for $C$. If the real value of the expression is retained and simplified, the result is equation 28 .

\section{References}

1. Vibration Analysis, W. B. Buchanan. Elec. Engg. (A.I.e.e. Trans.), v. 53, Nov. 1934, p. 1478-85.

2. Overcoming Vibration in Transmission Cables, G. H. Stockbridge. Electrical World, v. 86, Dec. 26, 1925, p. 1304-05.

3. Resistance of A Body Moving in A Fluid, T. von Kármán and H. Rubach. Gesell. Wiss. Gottingen, Nach., Math. Phys. Klasse 5, 1911, p. 509-17.

4. Aeolian Tones, J. W. S. Rayleigh. Scientific Papers (a book), Cambridge University Press, London, 1920, v. 6, p. 315-25.

5. Notes on the Vibration of Transmission Line Conductors, Theodore Varney. A.I.E.E. Trans., v. 45, 1926, p. 791-5.

6. Experiments on Cylindirs Oscillating in a Stream or Water, A. Thom. Phil. Mag., v. 12, series 7, July-Dec. 1931, p. 490-503.

7. Vibration of Overhead Transmission Lines, R. A. Monroe and R. L. Templin. A.I.E.E. Trans., v. 51, December 1932, p. 1059-73.

8. Hydrodynames (a book), Horace Lamb. Cambridge University Press, London, 1932.

\section{Better Visibility Needed on Highways at Night}

\begin{abstract}
More than half of the highway fatalities occur during the hours of darkness with a quarter of the daytime traffic volume. This is significant of conditions beyond the control of driver or pedestrian. Campaigns for education of driver and pedestrian, to be effective both day and night, must be accompanied by measures to improve night visibility, such as systems of fixed lights along the highways. The use of automobile headlights is ineffective and unscientific. The financing of highway lighting service is a state and not a county or township function. It is a vital necessity for preservation of life and property on the highway, and may readily be financed by surplus funds from gasoline taxation.
\end{abstract}

\section{By L. A. S. WOOD* MEMBER A.I.E.E.}

Westinghouse Elec. \& Mrg. Co., New York, N. Y.

\section{5}

EW PEOPLE in fields closely related to the problem of public safety, even in fields dealing directly with the problems of traffic safety, have realized the vital relationship of darkness with death. Few people have realized that existing traffic hazards remain unchanged throughout the 24 hours of the day, except that darkness comes down like a veil over all hazards once every day-comes down to confuse and blind the driver and pedestrian-comes down to reduce the time allowance for life saving reflexes-comes down to subject the eye to conditions with which it cannot cope-comes down, in fact, to increase fourfold the lethal potentialities of all other traffic hazards.

Proof, if proof be needed, that those responsible for public safety do not appreciate the relationship between highway darkness and death is found in press and radio campaigns to educate the driver and pedestrian and to enforce traffic regulations, with never a suggestion for the elimination of the greater hazard of darkness.

A paper recommended for publication by the A.I.E.E. committee on production and application of light, and scheduled for discussion at the A.I.E.E. summer convention, Pasadena, Calif., June 22-26, 1936. Manuscript submitted April 7, 1936; released for publication May 6,1936 . An address embracing the substance of this paper was presented at the A.I.E.E. North Eastern District meeting, New Haven, Conn., May 6-8, 1936 .

* President, Illuminating Engineering Society. 